Research Institute for Advanced Computer Science NASA Ames Research Center

\title{
A Review of \\ High-Order and Optimized Finite-Difference Methods for Simulating Linear Wave Phenomena
}

\author{
David W. Zingg \\ University of Toronto Institute for Aerospace Studies
}

RIACS Technical Report 96.12

July 1996

Submitted to the 13th AIAA CFD Conference 
. 


\title{
A Review of High-Order and Optimized Finite-Difference Methods for Simulating Linear Wave Phenomena
}

\author{
David W. Zingg \\ University of Toronto Institute for Aerospace Studies
}

The Research Institute for Advanced Computer Science is operated by Universities Space Research Association, The American City Building, Suite 212, Columbia, MD 21044, (410)730-2656

Work reported herein was supported by the National Aeronautics and Space Administration under Contract NAS 2-13721 to the Universities Space Research Association (USRA). 
. 


\section{Abstract}

This paper presents a review of high-order and optimized finite-difference methods for numerically simulating the propagation and scattering of linear waves, such as electromagnetic, acoustic, or elastic waves. The spatial operators reviewed include compact schemes, noncompact schemes, schemes on staggered grids, and schemes which are optimized to produce specific characteristics. The time-marching methods discussed include Runge-Kutta methods, Adams-Bashforth methods, and the leapfrog method. In addition, the following fourthorder fully-discrete finite-difference methods are considered: a one-step implicit scheme with a three-point spatial stencil, a one-step explicit scheme with a five-point spatial stencil, and a two-step explicit scheme with a five-point spatial stencil. For each method studied, the number of grid points per wavelength required for accurate simulation of wave propagation over large distances is presented. Recommendations are made with respect to the suitability of the methods for specific problems and practical aspects of their use, such as appropriate Courant numbers and grid densities. Avenues for future research are suggested.

\section{Introduction}

Numerical simulation can play an important role in the context of engineering design and in improving our understanding of complex systems. The subject of wave phenomena, including electromagnetic, elastic, and acoustic waves, is a promising area of application for such simulations. Lighthill [1] and Taflove [2] discuss the prospects for computational aeroacoustics and electromagnetics, respectively. The computational requirements of accurate simulations of the propagation and scattering of waves can be high, particularly if the size of the geometry under study is much larger than the wavelength. Consequently, there has been considerable recent effort directed towards improving the efficiency of numerical methods for simulating wave phenomena.

In electromagnetics, the most popular approach to the numerical solution of the timedomain Maxwell equations for numerous applications has been the algorithm of Yee [3], which was named the finite-difference time-domain (FDTD) method by Taflove [4]. This algorithm combines second-order centered differences on a staggered grid in space with second-order staggered leapfrog time marching. Its main attributes are its very low cost per grid node and lack of dissipative error. Yee's method is often applied using Cartesian grids, with a special tr atment of curved boundaries [5]. Extension to curvilinear grids was carried out by Fusco

[. Madsen and Ziolowski [7] put the method into a finite-volume framework applicable t unstructured grids. Vinokur and Yarrow [8,9] developed a related finite-surface method with advantages at boundaries and grid singularities. 
Other methods which have been successfully applied to the time-domain Maxwell equations include the upwind Lax-Wendroff approach used by Shankar [10], the characteristicbased fractional-step method of Shang [11], and the finite-element method of Cangellaris et al. [12] Although all of these second-order methods have been used with a great deal of success, they are efficient only for geometries of moderate electrical size, on the order of twenty wavelengths or less. For wave propagation over longer distances, the grid resolution requirements of second-order methods can become excessive, leading to impractical $\mathrm{CPU}$ and memory requirements. This has motivated the development of higher-order methods which produce smaller errors for a given grid resolution, such as the extension of Yee's method to fourth-order in space [2] and the methods of Liu [13], Shang [14], and Zingg et al. $[15,16,17,18]$

In seismology, the need for higher-order methods has been recognized for some time. Alford et al. [19], Marfurt [20], and Dablain [21] present higher-order algorithms for the elastic wave equation. Similarly, higher-order finite-difference methods have been developed for acoustic applications by Gottlieb and Turkel [22], Cohen and Joly [23], and Davis [24]. It can be advantageous to modify the coefficients of a potentially higher-order method, thereby lowering the order of accuracy, to produce reduced errors over a range of wavenumbers. This approach was first proposed by Vichnevetsky and De Schutter [25] and later studied in more detail by Holberg [26] and Lele [27]. Haras and Ta'asan [28] and later Kim and Lee [29] further refined the optimization technique used by Lele. The papers by Holberg and Lele spawned a number of optimized schemes, including those presented in Refs. [15], [16] and [30] to [34].

Numerical errors arise from both the spatial and the temporal discretization. They include both phase and amplitude errors, which depend on the wavenumber, the grid spacing, the Courant number, and the direction of propagation relative to the grid. The dependence of the phase speed on the wavenumber results in numerical dispersion. This paper presents the grid resolution required to achieve a specific level of accuracy as a function of the propagation distance expressed in terms of the wavelength for several high-order and optimized methods. Emphasis is on methods requiring under 30 grid points per wavelength $(P P W)$ for accurate simulations with propagation distances of 200 wavelengths. The errors are calculated using Fourier analysis $[35,36,16]$. The purpose is to aid in selecting and using a scheme for a given application, and to provide direction for future research.

In addition to the accuracy of the interior differencing scheme, there are several other important issues in simulating wave phenomena. High-order and optimized methods often have a large spatial stencil which cannot be used at boundaries. This necessitates the use of numerical boundary schemes (NBS's) which must be appropriately accurate and stable. These can be difficult to obtain, and thus this represents the most significant obstacle in the use of higher-order methods. Recent progress is reported in Refs. [37] to [40]. Another important consideration is the boundary condition at the outer boundary of the domain, which inevitably causes spurious reflection. Since this does not decrease with the grid spacing, it can become the limiting factor in determining the accuracy of a simulation. Important early contributions to the development of non-reflecting boundary conditions are given by 
Engquist and Majda [41] and Bayliss and Turkel [42]. Mur [43] implemented the EngquistMajda approach for the time-domain Maxwell equations. Moore et al. [44] present a detailed comparison of non-reflecting boundary conditions available at that time, again in an electromagnetic context. A review of developments up to 1991 is given by Givoli [45]. Progress since that time includes the approaches of Tam and Webb [46] and Berenger [47], both of which show considerable promise. A more recent comparison, in an aeroacoustic context, is presented by Hixon et al. [48]

Another important consideration is the choice of a gridding strategy. Although unstructured grids have been used [7], Cartesian and curvilinear grids are generally preferred. Uniform Cartesian grids are used, for example, by Taflove [2] and Tam [49], while body-fitted curvilinear grids are favoured by Shankar et al. [10], Jurgens and Zingg [17, 18], and others. The Cartesian approach produces two significant advantages in the interior of the domain. Virtually all numerical methods are most accurate on a uniform grid, and furthermore, the grid size can be chosen to give the Courant number which produces the smallest errors for the scheme used. In addition, the need to deal with the metrics of a curvilinear coordinate transformation leads to a substantial penalty in terms of both speed and memory. On the other hand, it is extremely difficult to develop stable and accurate boundary treatments for higher-order methods on Cartesian grids. Refs. [5] and [50] present progress in this area. Furthermore, some geometries, such as a curved surface with a thin coating, are not well suited to Cartesian grids. Clearly, the choice of a gridding strategy is problem dependent.

\section{Finite-Difference Methods Compared}

In this section, we present the various finite-difference methods in the context of the linear advection equation given by

$$
\frac{\partial u}{\partial t}+a \frac{\partial u}{\partial x}=0
$$

where $u$ is a scalar quantity propagating with speed $a$, which is real and positive. The spatial difference operators are thus approximations to $\partial / \partial x$. Extension to systems of equations and multidimensions is not discussed. The time-marching methods are presented as applied to a scalar ordinary differential equation of the form

$$
\frac{d u}{d t}=f(u, t)
$$

The fully-discrete schemes are given as applied to eq. 1 itself. The coefficients of all of the schemes studied are given in the Appendix. 


\section{Spatial Difference Operators}

On a uniform grid with $x_{j}=j \Delta x$ and $u_{j}=u\left(x_{j}\right)$, compact centered-difference schemes of up to tenth order can be represented by the following formula:

$$
\begin{aligned}
& \beta\left(\delta_{x} u\right)_{j-2}+\alpha\left(\delta_{x} u\right)_{j-1}+\left(\delta_{x} u\right)_{j}+\alpha\left(\delta_{x} u\right)_{j+1}+\beta\left(\delta_{x} u\right)_{j+2} \\
= & \frac{1}{2 \Delta x}\left[\frac{c}{3}\left(u_{j+3}-u_{j-3}\right)+\frac{b}{2}\left(u_{j+2}-u_{j-2}\right)+a\left(u_{j+1}-u_{j-1}\right)\right]
\end{aligned}
$$

where $\left(\delta_{x} u\right)_{j}$ is an approximation to $\partial / \partial x$ at node $j$. These schemes produce no dissipative error. Noncompact schemes of up to sixth order are obtained with $\beta=\delta=0$.

Haras and Ta'asan [28] revisited the compact schemes of Lele [27] based on eq. 3 using a more sophisticated optimization procedure. They developed tridiagonal schemes $(\beta=$ 0 ) and tridiagonal schemes with a five-point stencil $(\beta=c=0)$ as well. In each case, Haras and Ta'asan present several methods which result from different parameters in the optimization procedure. In the comparisons below, we will include only those schemes which are best according to our criterion, i.e., those schemes which require the fewest grid points per wavelength for accurate wave propagation over a distance of two hundred wavelengths.

The spatial operator of Zingg et al. $[15,16]$ is noncompact with a seven-point stencil. The operator is divided into an antisymmetric part given by

$$
\left(\delta_{x}^{a} u\right)_{j}=\frac{1}{\Delta x}\left[a_{3}\left(u_{j+3}-u_{j-3}\right)+a_{2}\left(u_{j+2}-u_{j-2}\right)+a_{1}\left(u_{j+1}-u_{j-1}\right)\right]
$$

and a symmetric part given by

$$
\left(\delta_{x}^{s} u\right)_{j}=\frac{1}{\Delta x}\left[d_{3}\left(u_{j+3}+u_{j-3}\right)+d_{2}\left(u_{j+2}+u_{j-2}\right)+d_{1}\left(u_{j+1}+u_{j-1}\right)+d_{0} u_{j}\right]
$$

The symmetric part provides dissipation of spurious high-wavenumber components of the solution and is required for stability of the time-marching method used, which will be presented in the next subsection. The magnitude of the dissipative component is chosen such that the dissipative error produced is comparable to the dispersive error. Refs. [15] and [16] include a maximum-order scheme and an optimized scheme. The optimized scheme was designed to minimize the maximum phase and amplitude errors for waves resolved with at least ten $P P W$. It is superior for distances of travel of up to 330 wavelengths. Hence we do not consider the maximum-order scheme here. Tam [31] has also developed an optimized scheme based on the antisymmetric operator given in eq. 4 .

Lockard et al. [30] present an optimized upwind-biased noncompact spatial difference operator based on an eight-point stencil. It can be written in the form

$$
\left(\delta_{x} u\right)_{j}=\frac{1}{\Delta x} \sum_{m=-4}^{3} a_{m} u_{j+m}
$$

The operator is optimized for waves resolved with at least seven points per wavelength and provides excellent accuracy up to about 340 wavelengths of travel. 
Differencing schemes on staggered grids are well suited to problems in which the time derivative of one variable depends on the spatial derivative of the other, and vice-versa. This is the case for the time-domain Maxwell equations or the Euler equations linearized about a reference state with zero velocity, for example. Centered staggered differencing schemes of up to sixth order can be written in the form

$$
\left(\delta_{x} u\right)_{j}=\frac{1}{\Delta x}\left[b_{1}\left(u_{j+1 / 2}-u_{j-1 / 2}\right)+b_{2}\left(u_{j+3 / 2}-u_{j-3 / 2}\right)+b_{3}\left(u_{j+5 / 2}-u_{j-5 / 2}\right)\right]
$$

\section{Time-Marching Methods}

There are many considerations involved in selecting a time-marching method, including efficiency, i.e., accuracy per unit computational effort, stability, and memory use. Since wave propagation problems are generally not stiff, explicit methods are appropriate. Thus the Adams-Bashforth and Runge-Kutta families are suitable candidates. Since a large portion of the computational effort is generally associated with evaluation of the derivative function, one can approximately assess efficiency by accounting for the number of derivative function evaluations per time step. Runge-Kutta methods require one derivative function evaluation per stage. Zingg and Chisholm [51] have shown that for linear ordinary differential equations (ODE's) with constant coefficients, Runge-Kutta methods of up to sixth-order (and probably arbitrary order) can be derived with a number of stages equal to the order. However, the memory requirements of the methods of order five and six are high. An alternative approach is to use low-storage multi-stage methods which are high-order for homogeneous linear ODE's but second-order otherwise [52]. Haras and Ta'asan [28] and Zingg et al. [15, 16] present five- and six-stage methods of this type, respectively. For example, when applied to eq. 2, the six-stage method of Refs. [15] and [16] is given by

$$
\begin{aligned}
u_{n+\alpha_{1}}^{(1)} & =u_{n}+h \alpha_{1} f_{n} \\
u_{n+\alpha_{2}}^{(2)} & =u_{n}+h \alpha_{2} f_{n+\alpha_{1}}^{(1)} \\
u_{n+\alpha_{3}}^{(3)} & =u_{n}+h \alpha_{3} f_{n+\alpha_{2}}^{(2)} \\
u_{n+\alpha_{4}}^{(4)} & =u_{n}+h \alpha_{4} f_{n+\alpha_{3}}^{(3)} \\
u_{n+\alpha_{5}}^{(5)} & =u_{n}+h \alpha_{5} f_{n+\alpha_{4}}^{(4)} \\
u_{n+1} & =u_{n}+h f_{n+\alpha_{5}}^{(5)}
\end{aligned}
$$

where $h=\Delta t$ is the time step, $t_{n}=n h, u_{n}=u\left(t_{n}\right)$, and

$$
f_{n+\alpha}^{(k)}=f\left(u_{n+\alpha}^{(k)}, t_{n}+\alpha h\right)
$$

The five-stage method of Ref. [28] is analogous. In their maximum-order form (for homogeneous ODE's), these methods are not stable for pure central differencing in space. Consequently, Haras and Ta'asan modified the coefficients of the five-stage scheme to produce stability, while Zingg et al.added some dissipation to their spatial operator. 
Since Adams-Bashforth methods require only one derivative function evaluation per time step, they are more efficient than Runge-Kutta methods of equal order. Unfortunately, Adams-Bashforth methods of order higher than four have extremely restrictive stability bounds which outweigh this advantage. Thus the time-marching methods we consider here are the fourth-order Adams-Bashforth method, the fourth-order Runge-Kutta method for linear ODE's given by Zingg and Chisholm [51], and the low-storage five- and six-stage methods described above. In terms of stability and Fourier error analysis, the fourth-order RungeKutta method of Ref. [51] is identical to the classical fourth-order Runge-Kutta method. With respect to memory requirements, the fourth-order Adams-Bashforth method requires five memory locations per dependent variable. The other methods considered require only two.

A natural choice of time-marching method for use with staggered spatial differencing is the second-order staggered leapfrog method, given by

$$
u_{n+1}=u_{n}+h f_{n+1 / 2}
$$

When used with a nondissipative spatial scheme, as in the FDTD method, the resulting fullydiscrete operator produces no dissipative error. Furthermore, the staggered leapfrog method generally produces a leading phase error, which can offset the phase lag usually produced by centered spatial differences. At specific Courant numbers and angles of propagation, the perfect-shift property can be obtained, leading to exact propagation for all wavenumbers. Although this has little practical significance, fully-discrete methods which possess this property are generally most accurate when used near the perfect-shift conditions.

\section{Simultaneous Space-Time Discretizations}

The schemes presented in this subsection approximate the spatial and temporal derivatives simultaneously. No intermediate semi-discrete form is produced. All of the schemes are fourth-order in time and space.

The scheme of Davis [24] is a nondissipative implicit scheme with a three-point spatial stencil. When applied to the linear advection equation, it is given by

$$
a_{0} u_{j}^{n+1}+a_{1} u_{j-1}^{n+1}+a_{2} u_{j+1}^{n+1}=b_{0} u_{j}^{n}+b_{1} u_{j-1}^{n}+b_{2} u_{j+1}^{n}
$$

with $a_{0}=b_{0}, a_{1}=b_{2}$, and $a_{2}=b_{1}$, where $u_{j}^{n}=u\left(x_{j}, t_{n}\right)$. Being implicit, this scheme requires somewhat more computational expense than explicit noncompact schemes. However, it achieves fourth-order accuracy with a three-point spatial stencil.

The scheme of Gottlieb and Turkel [22] is an extension of the Lax-Wendroff approach to fourth-order. It is an explicit one-step scheme but requires a five-point stencil. When applied to the linear advection equation, it is given by

$$
\begin{aligned}
u_{j}^{(1)} & =u_{j}^{n}+\frac{a h}{6 \Delta x}\left(u_{j+2}^{n}-8 u_{j+1}^{n}+7 u_{j}^{n}\right) \\
u_{j}^{n+1} & =\frac{1}{2}\left(u_{j}^{n}+u_{j}^{(1)}\right)+\frac{a h}{12 \Delta x}\left(-u_{j-2}^{(1)}+8 u_{j-1}^{(1)}-7 u_{j}^{(1)}\right)
\end{aligned}
$$


This method is dissipative and has received considerable use in nonlinear problems.

Finally, we consider the following two-step explicit scheme [53]:

$$
u_{j}^{n+1}=u_{j}^{n-1}-2 a h\left(\delta_{x}^{(4)}\right)_{j}^{n}-\frac{h^{3} a^{3}}{3}\left(\delta_{x x x}^{(2)}\right)_{j}^{n}
$$

where $\delta_{x}^{(4)}$ is a fourth-order centered difference approximation to a first derivative and $\delta_{x x x}^{(2)}$ is a second-order centered approximation to a third derivative. Both of the operators on the right-hand side require a five-point stencil, as follows:

$$
\begin{aligned}
\left(\delta_{x}^{(4)}\right)_{j} & =\frac{1}{12 \Delta x}\left(u_{j-2}-8 u_{j-1}+8 u_{j+1}-u_{j+2}\right) \\
\left(\delta_{x x x}^{(2)}\right)_{j} & =\frac{1}{2 \Delta x}\left(-u_{j-2}+2 u_{j-1}-2 u_{j+1}+u_{j+2}\right)
\end{aligned}
$$

This scheme is derived using simple Taylor series expansions and is nondissipative. Related methods for the second-order wave equation are presented by Cohen and Joly [23] and Shubin and Bell [54].

\section{Fourier Error Analysis}

Fourier analysis provides a simple means of determining the errors produced by finitedifference methods in the absence of boundary conditions. In one dimension, these are functions of the wavenumber, $\kappa$, the grid spacing, and the Courant number, $C=a h / \Delta x$. In multidimensions, the errors further depend on the direction of propagation relative to the grid. The error analysis is based on the principal root, $\sigma(z, C)$, where $z=\kappa \Delta x$, i.e., $z=2 \pi / P P W$. The spurious roots are required for stability analysis. The local amplitude and phase errors are, respectively,

$$
\begin{gathered}
e r_{a}=|\sigma|-1 \\
e r_{p}=-\frac{\phi}{z C}-1
\end{gathered}
$$

where $\phi=\arctan \left(\sigma_{i} / \sigma_{r}\right)$ and $\sigma_{r}$ and $\sigma_{i}$ are the real and imaginary parts of $\sigma$, respectively. Our criterion for comparing schemes is based on the global amplitude and phase errors, which are

$$
\begin{aligned}
& E r_{a}=\left.|1-| \sigma\right|^{P P W n_{w} / C} \mid \\
& E r_{p}=n_{w}\left|\frac{P P W \phi}{C}+2 \pi\right|
\end{aligned}
$$


where $n_{w}$ is the number of wavelengths travelled. Using the above formulas with a very small Courant number gives the errors for the spatial operator alone. In the figures below, the various methods are compared in terms of the $P P W$ required to keep both global phase and amplitude errors below 0.1 as a function of the number of wavelengths travelled.

The error from a spatial discretization is often plotted in terms of the modified wavenumber. Plots of the local phase and amplitude errors can be much more revealing and provide a stronger physical connection. The global errors are even easier to interpret. In studying the dependence of the $P P W$ requirements on the number of wavelengths travelled, the emphasis is on the wavenumber present in the simulation which is most poorly resolved among those wavenumbers which are deemed to be significant. This is a reasonable measure for selecting a grid density. Furthermore, this method of presentation clearly reveals the implications of optimization.

Time-marching methods can be analyzed in isolation by applying them to the following linear homogeneous ODE:

$$
\frac{d u}{d t}=\lambda u
$$

In order to assess their accuracy for wave propagation, we consider only pure imaginary values of $\lambda$, i.e., $\lambda=i \omega$ with $\omega$ real. The local amplitude and phase errors are determined from the principal root, $\sigma(\lambda h)$, as follows:

$$
\begin{aligned}
& e r_{a}=|\sigma|-1 \\
& \epsilon r_{p}=-\frac{\arctan \left(\sigma_{i} / \sigma_{r}\right)}{\omega h}+1
\end{aligned}
$$

\section{Results}

\section{Spatial Difference Operators}

In this section, we consider the errors produced by the spatial operators alone. Figure 1 shows the grid resolution requirements for second-, fourth-, and sixth-order noncompact centered difference schemes. Since these spatial operators are nondissipative, the grid resolution requirements are determined from the global phase error. The requirements of the secondorder scheme are clearly excessive. While more accurate second-order discretizations are available, such as the FDTD scheme or the upwind leapfrog scheme [55], none of these meets our criterion of $30 \mathrm{PPW}$ for 200 wavelengths of travel except under specific conditions [56].

The $P P W$ requirements for compact centered difference schemes of up to tenth order are shown in Figure 2. The tenth-order scheme requires the solution of a pentadiagonal system of equations. The remaining schemes lead to tridiagonal systems. The compact schemes are considerably more accurate than the noncompact schemes of equivalent order. 


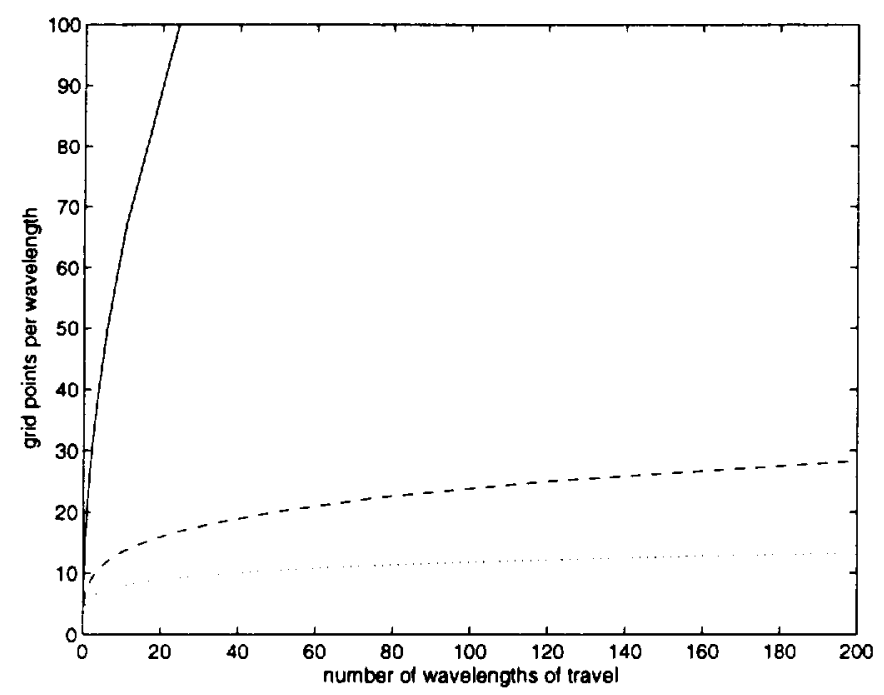

Figure 1: Grid resolution requirements for second-order (-), fourth-order (- - ) , and sixthorder $(\cdots)$ noncompact centered spatial differences.

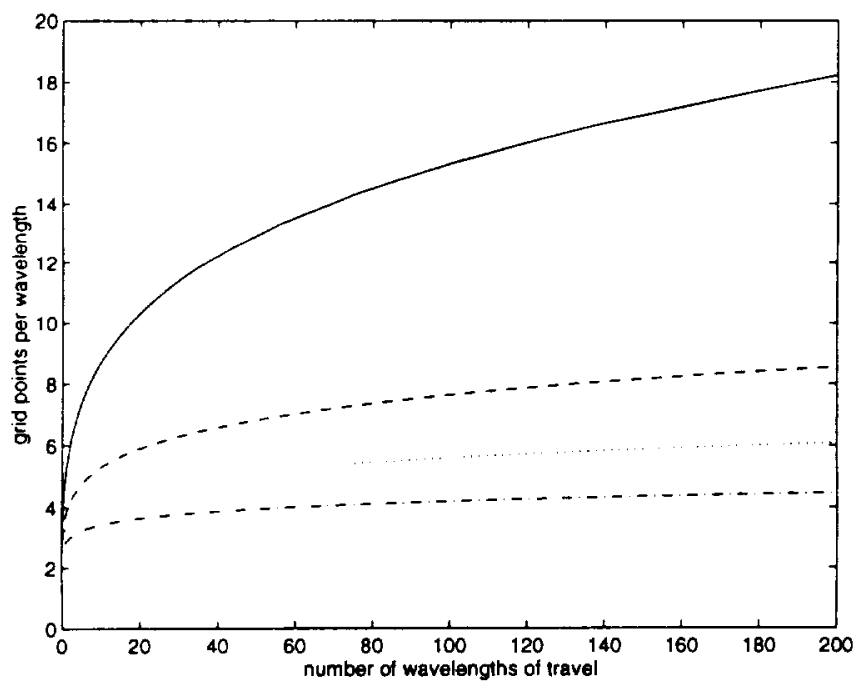

Figure 2: Grid resolution requirements for fourth-order (-), sixth-order (- - ), eighth-order $(\cdots)$, and tenth-order $(-\cdot-)$ compact centered spatial differences. 


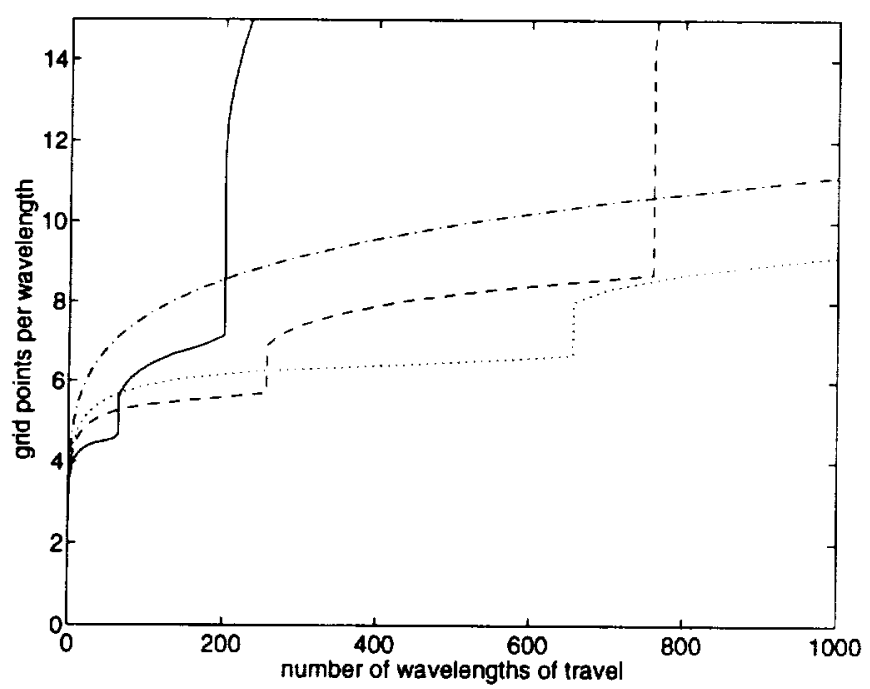

Figure 3: Grid resolution requirements for the tridiagonal spatial operators with 5-point right-hand stencil of Haras and Ta'asan: scheme A $(-)$, scheme B (- - ), scheme C ( . - ) sixth-order scheme $(-\cdot-)$.

We next demonstrate the tradeoffs associated with optimization, using the tridiagonal five-point operators $(\beta=c=0)$ of Haras and Ta'asan as an example. Figure 3 shows the behavior of three different optimized schemes given in Ref. [28] as well as the maximumorder scheme which can be obtained using this operator, which is sixth-order. The scheme denoted "A" is superior up to a distance of travel of about 60 wavelengths. Scheme B is superior for distances up to 250 wavelengths while scheme $C$ is preferable for even longer distances. Such behavior is typical of optimized schemes. Aggressive optimization (as in the case of scheme A) leads to excellent performance for small distances of travel but poor performance for longer distances. In this paper we concentrate on distances of travel up to 200 wavelengths. Hence we consider only scheme $B$ further.

Figure 4 shows the $P P W$ required by the optimized schemes of Haras and Ta'asan for distances of travel up to two hundred wavelengths. In each case, the best scheme presented by Haras and Ta'asan for this distance of travel is shown, as discussed above. The pentadiagonal scheme requires about $3.7 P P W$ for two hundred wavelengths of travel while the tridiagonal seven-point scheme requires 4.5 and the tridiagonal five-point scheme requires 5.7. The computational effort is roughly proportional to $P P W^{d+1}$, where $d$ is the number of dimensions. Therefore the increased cost per grid node of the pentadiagonal scheme is probably not justified. However, the tridiagonal seven-point scheme is likely to be more efficient than the tridiagonal five-point scheme.

The noncompact optimized schemes of Lockard et al., Zingg et al., and Tam are compared with sixth-order centered differences in Figure 5. For the scheme of Lockard et al., the PPW 


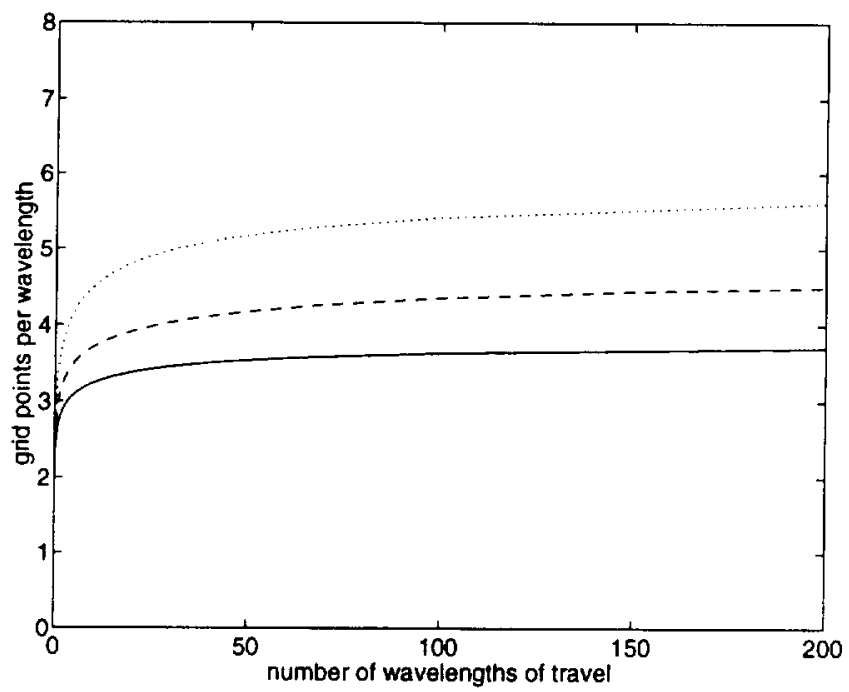

Figure 4: Grid resolution requirements for the spatial operators of Haras and Ta'asan: pentadiagonal operator with 7-point right-hand stencil (-), tridiagonal operator with 7-point right-hand stencil (- - ), tridiagonal operator with 5-point right-hand stencil $(\cdots)$.

requirements are determined by the amplitude error. For the scheme of Zingg et al., the phase and amplitude errors produce roughly equivalent grid resolution requirements over this distance. Since Tam's scheme is nondissipative, its $P P W$ requirements are determined by the phase error. The figure shows that Tam's scheme has been optimized for fairly small distances of travel, leading to excellent performance for less than roughly 14 wavelengths of travel. The scheme of Lockard et al. requires roughly $8 P P W$ for two hundred wavelengths of travel while that of Zingg et al. requires over $9 P P W$. This again is probably sufficient to compensate for the increased expense of the Lockard scheme, which uses an eight-point stencil versus seven for the Zingg scheme. However, it can be difficult to find stable and accurate numerical boundary schemes for use near boundaries when the spatial stencil is large. For the scheme of Zingg et al., such numerical boundary schemes are given in Ref. [17]. Comparison of these two schemes with those of Haras and Ta'asan is difficult. Although the compact schemes have significantly reduced grid resolution requirements, they also require considerably greater computational effort per grid node. Numerical experiments are required to determine which approach is more efficient.

Figure 6 shows the $P P W$ requirements of the staggered spatial operators. In each case, the staggered schemes are much more accurate than their nonstaggered counterparts of equivalent order. The grid requirements of the second-order scheme are again excessive. However, when used with staggered leapfrog time marching (the FDTD scheme), better results can be obtained. Also shown in Figure 6 is an optimized scheme with $b_{3}=103 / 19200$, $b_{2}=-1315 / 19200$, and $b_{1}=22630 / 19200$, which produces excellent performance for up to 


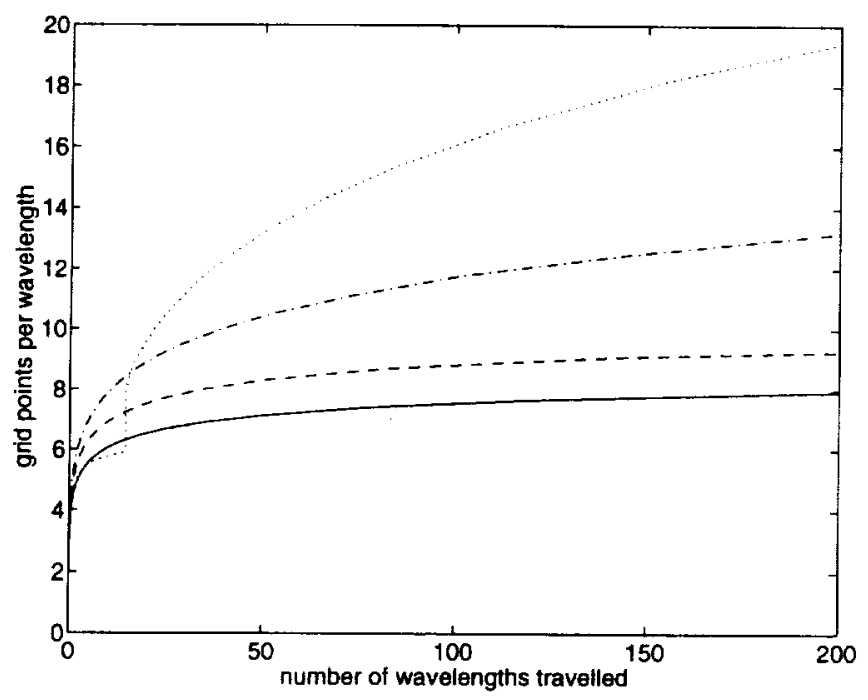

Figure 5: Grid resolution requirements for the spatial operators of Lockard et al. (-), Zingg et al. (--), Tam ( $\cdots)$, and sixth-order centered differences $(-\cdots)$.

200 wavelengths of travel.

\section{Time-Marching Methods}

Figures 7 and 8 show the amplitude and phase errors produced by the four time-marching methods under consideration. The five- and six-stage methods shown are the maximum-order versions rather than the optimized methods, which are discussed in the next subsection. In order to account for the number of stages in the Runge-Kutta methods, the errors are plotted versus $\omega h / p$, where $p$ is the number of stages. Hence the errors shown are for approximately equal computational effort. Since the time step of a $p$-stage scheme is thus $p$ times larger than that of a single-stage scheme, the amplitude error shown is $|\sigma|^{1 / p}-1$.

The figures show that the phase errors produced by these methods are larger than the amplitude errors. Each increase in the order of the Runge-Kutta method produces an increase in accuracy even though the extra work has been accounted for. The fourth-order AdamsBashforth method is much more accurate than the fourth-order Runge-Kutta method. It produces the lowest amplitude error of the methods considered and phase error comparable to the five-stage Runge-Kutta method.

In order to compare time-marching methods properly, one must consider the spatial discretization to be used. For each combination of a spatial discretization and a timemarching method, there is a Courant number which minimizes the computational work to achieve a given standard of accuracy for a specified distance of travel. The computational 


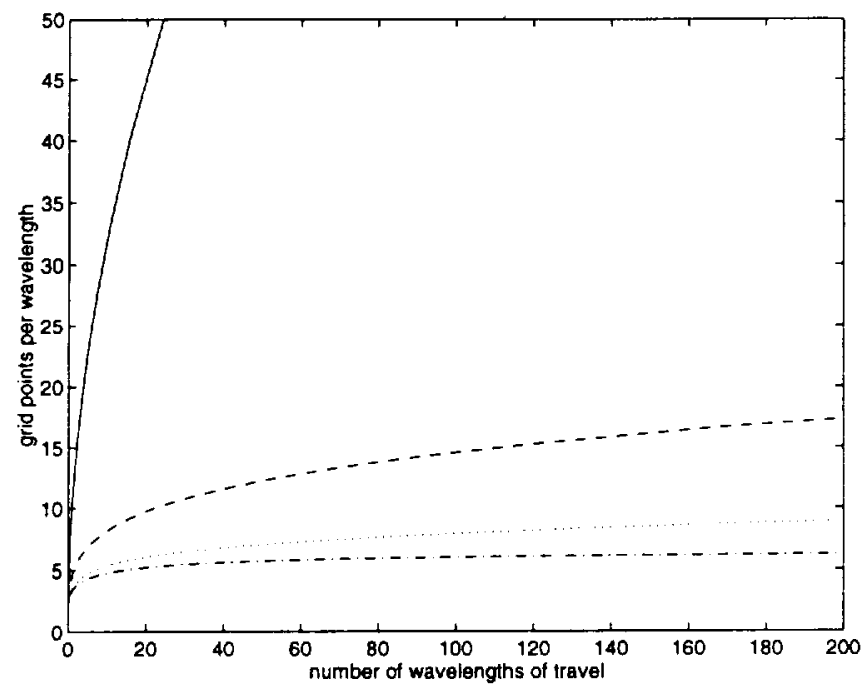

Figure 6: Grid resolution requirements for second-order (-), fourth-order (- - ), sixth-order $(\cdots)$, and optimized $(-\cdot-)$ centered spatial differences on a staggered grid.

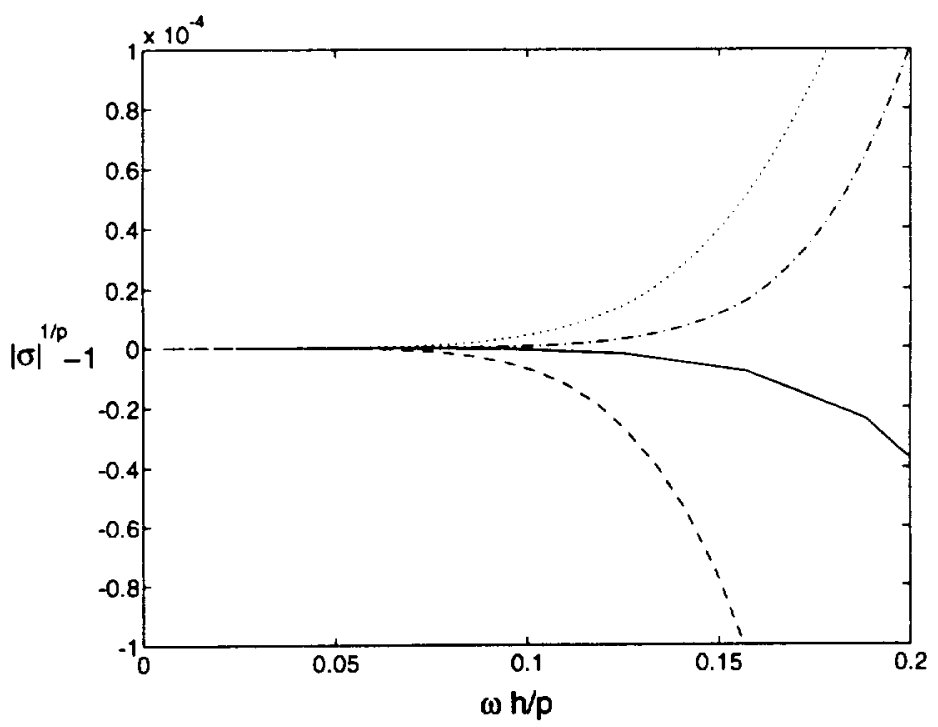

Figure 7: Amplitude error produced by the fourth-order Adams-Bashforth method (-), the fourth-order Runge-Kutta method (- -), the five-stage Runge-Kutta method ( $\cdots)$, and the six-stage Runge-Kutta method $(-\cdot-)$. 


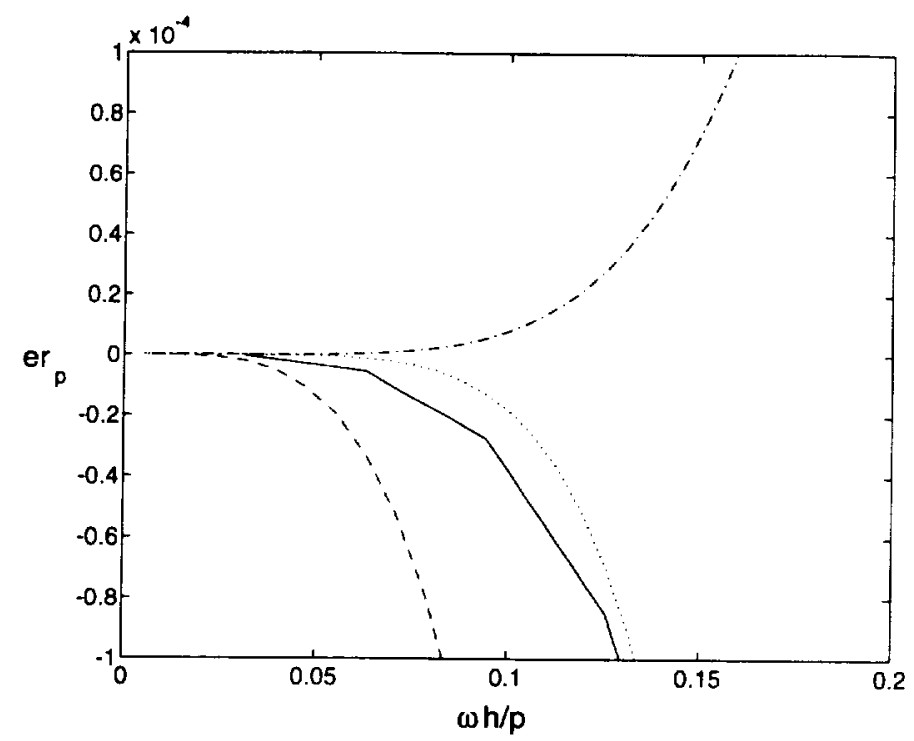

Figure 8: Phase error produced by the fourth-order Adams-Bashforth method (-), the fourth-order Runge-Kutta method ( - - ), the five-stage Runge-Kutta method ( ...), and the six-stage Runge-Kutta method (- - - ).

work is proportional to

$$
F=\frac{p}{C} P P W^{d+1}
$$

where $p$ is again the number of stages and $d$ the number of dimensions. For example, with fourth-order centered differences in space the optimum Courant number for the fourthorder Runge Kutta method in two dimensions is about 1.25 for two hundred wavelengths of travel and global errors less than 0.1 . With this spatial operator, the optimum Courant number for the Adams-Bashforth method is determined by its stability bound, which for pure imaginary $\lambda$ is roughly 0.43 . With fourth-order centered differences in two dimensions, the resulting Courant number is about 0.21 . The value of $F$ produced at this Courant number is slightly higher than that produced using the fourth-order Runge-Kutta method at its optimum Courant number. Since the fourth-order Runge-Kutta method also requires less memory than the fourth-order Adams-Bashforth method, it is clearly the preferred method for use with fourth-order centered differences in space.

With sixth-order centered differences in space, the comparison changes. The optimum Courant number for the fourth-order Runge-Kutta method is reduced to roughly $2 / 3$ while that for the fourth-order Adams-Bashforth method remains stability limited, leading to a Courant number of roughly 0.19 . In this case the value of $F$ produced by the AdamsBashforth method is less than $80 \%$ of that for the Runge-Kutta method. Thus the comparison is more complicated, as one must weigh the increased efficiency of the Adams-Bashforth method against the reduced memory requirements of the Runge-Kutta method.

The difficulty with the maximum-order five- and six-stage Runge-Kutta methods is that 
they are unstable for pure imaginary $\lambda$, as shown in Figure 7 . We consider these schemes further below.

\section{Combined Space-Time Discretizations}

Haras and Ta'asan modified the coefficients of the five-stage Runge-Kutta method to produce stability for pure imaginary $\lambda$ while maintaining second-order accuracy and optimized error behavior. The methods are designed for $C=0.9$. Figure 9 shows the grid resolution requirements of the three spatial operators of Haras and Ta'asan compared in Figure 4 in combination with their five-stage time-marching method at a Courant number of 0.9 . All three schemes require between 7 and $8 P P W$ for two hundred wavelengths of travel. The advantage of the more accurate spatial operators has been lost. Either a lower Courant number or a more accurate time-marching method should be used.

As a result of the dissipation in the spatial operator, the six-stage time-marching method of Zingg et al. is stable up to a Courant number a little greater than unity in two dimensions. The grid requirements for the combined space-time discretization at a Courant number of unity are shown in Figure 9. Comparison with Figure 5 shows that the time-marching method introduces very little error compared to the spatial differencing. Tam [31] uses an optimized four-step Adams-Bashforth method in conjunction with his spatial operator. It produces little error for Courant numbers less than 0.3. In both cases, optimization of the time-marching method has a much smaller impact than optimization of the spatial operator.

Figure 10 shows the $P P W$ requirements of the fourth-order staggered spatial difference operator combined with staggered leapfrog time marching. As the Courant number is increased from 0.001 to 0.1 , the $P P W$ requirements decrease, since the error from the time marching method is of opposite sign to that of the spatial operator. However, as the Courant number is further increased, the error from the second-order time-marching method begins to dominate. Excellent performance for 200 wavelengths of travel is obtained for Courant numbers up to 0.1 .

Results for the method of Davis are shown in Figure 11. Since the method is nondissipative, the $P P W$ requirements are determined from the phase error. This scheme is unconditionally stable. For Courant numbers under 1.5, less than $19 P P W$ are required for two hundred wavelengths of travel. While this is quite good, much better than many schemes, it is probably not sufficient to justify the additional computational effort associated with an implicit scheme.

Figure 12 shows the grid resolution requirements for the Gottlieb-Turkel scheme, which is stable up to a Courant number of $2 / 3$ in one dimension. This scheme is very robust and has received extensive use in nonlinear applications. However, high accuracy is obtained only for Courant numbers less than about 0.13 . Less than $29 P P W$ are required to propagate a wave two hundred wavelengths with Courant numbers below this value. Although the cost per grid node is reasonably low, these $P P W$ requirements are too high for efficient simulation over such distances.

Finally, the grid requirements of the nondissipative two-step explicit scheme, eq. 12, 


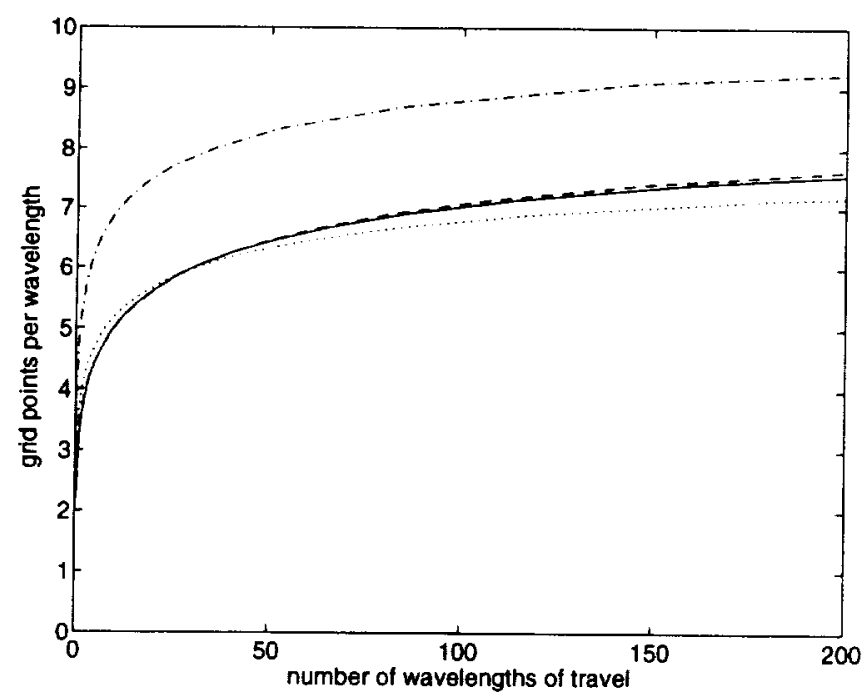

Figure 9: Grid resolution requirements for the spatial and temporal operators of Haras and Ta'asan at a Courant number of 0.9: pentadiagonal operator with 7-point right-hand stencil (-), tridiagonal operator with 7-point right-hand stencil (- - ), tridiagonal operator with 5 -point right-hand stencil $(\cdots)$; spatial and temporal operators of Zingg et al. at a Courant number of $1(-\cdot-)$.

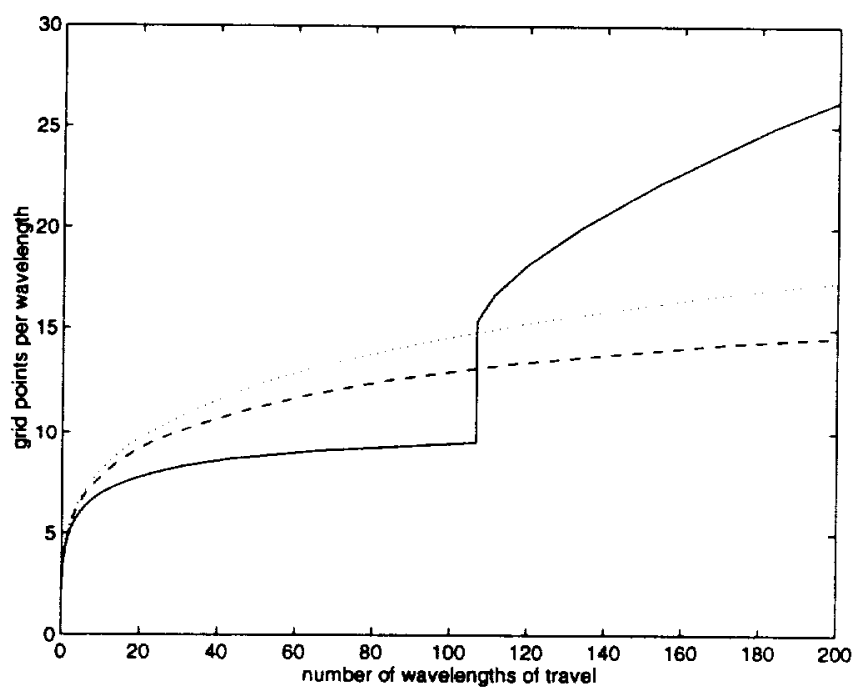

Figure 10: Grid resolution requirements for fourth-order centered differences on a staggered grid coupled with staggered leapfrog time marching at Courant numbers of $0.2(-), 0.1$ ($--)$, and $0.001(\cdots)$. 


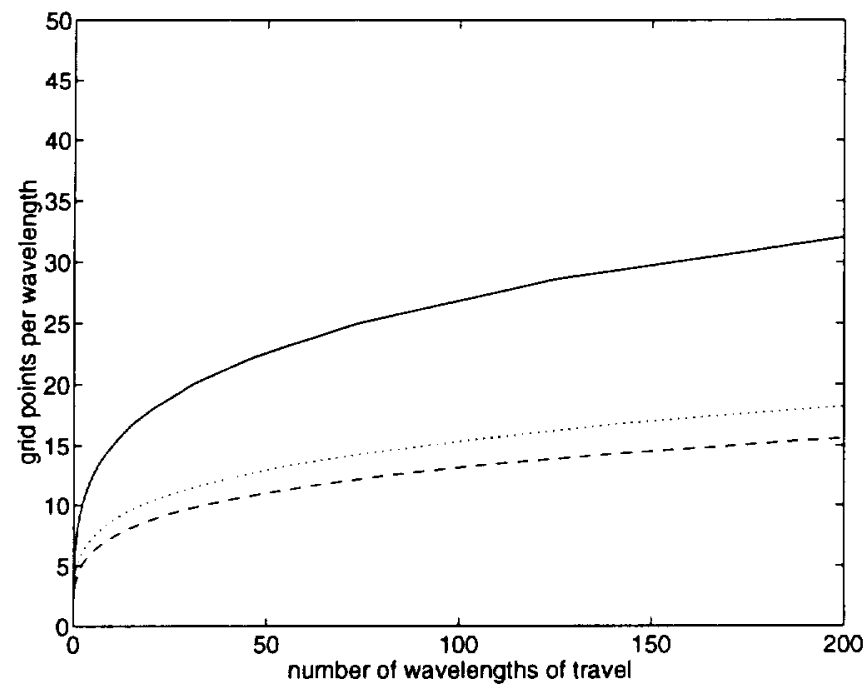

Figure 11: Grid resolution requirements for the scheme of Davis at Courant numbers of 3 $(-), 1.5(--)$, and $0.01(\cdots)$.

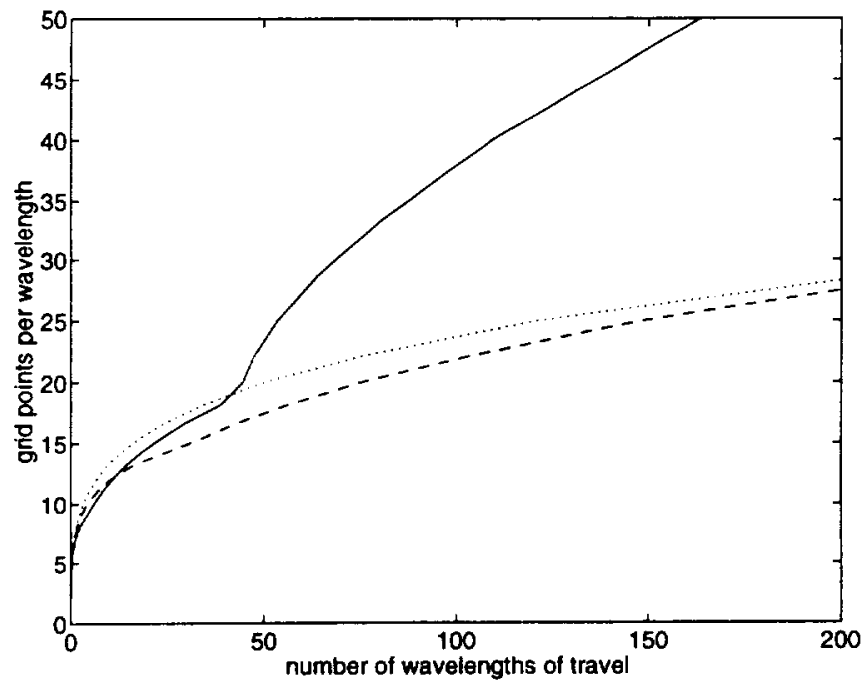

Figure 12: Grid resolution requirements for the scheme of Gottlieb and Turkel at Courant numbers of $0.2(-), 0.13(--)$, and $0.01(\cdots)$. 


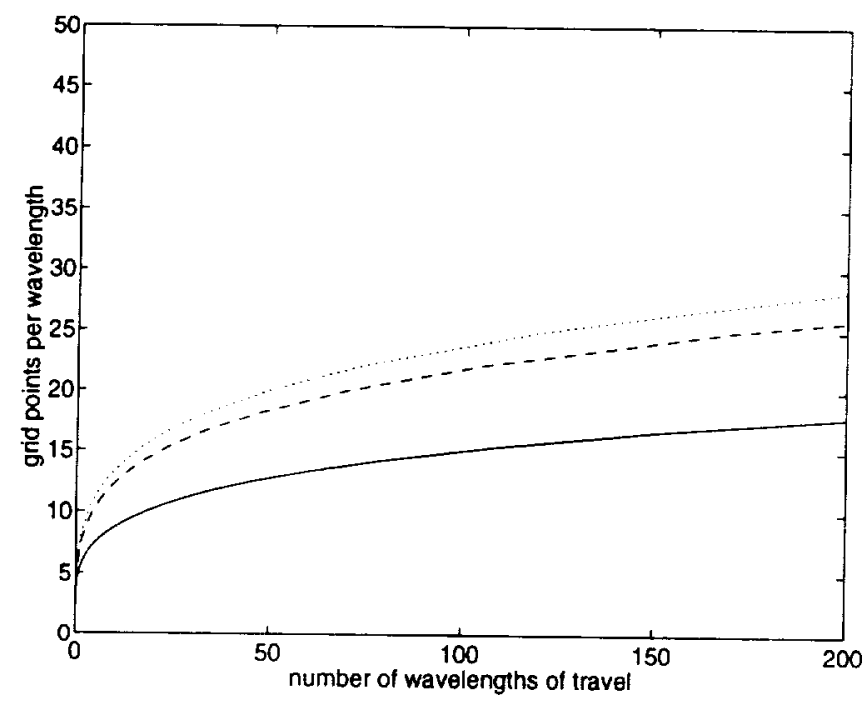

Figure 13: Grid resolution requirements for the two-step explicit scheme (eq. 10) at Courant numbers of $0.9(-), 0.5(\cdots)$, and $0.01(\cdots)$.

are shown in Figure 13. This scheme is stable up to a Courant number of unity in one dimension. It has the perfect shift property at a Courant number of unity. The phase error increases as the Courant number is reduced. Less than $29 P P W$ are required for all stable Courant numbers. Since it provides reasonably low error at Courant numbers up to unity, this scheme has a very low cost per grid node, substantially lower than the Gottlieb-Turkel scheme. However, the PPW requirements are again much higher than some of the other schemes considered.

\section{Discussion}

The grid requirements presented can be used to determine a suitable grid density and Courant number when applying a method to a specific problem. Estimates of the shortest wavelength of interest and the distance of travel are required. However, long distances of travel are generally associated with multiple reflections. Therefore, the accuracy of a simulation is strongly dependent on the accuracy of the boundary condition formulation as well as the interior finite-difference scheme. This represents a major obstacle in the application of highaccuracy schemes such as those of Haras and Ta'asan.

The results show that optimized schemes provide a significant advantage over their maximum-order counterparts. However, if optimized too aggressively, they perform poorly for longer distances of travel. If a waveform has significant low wavenumber content, as 
in the case of a Gaussian pulse, maximum-order schemes can produce smaller errors than optimized schemes. Furthermore, numerical boundary schemes affect the relative accuracy of optimized and maximum-order schemes.

The spatial operators of Haras and Ta'asan, Lockard et al., and Zingg et al. all provide adequate accuracy for two hundred wavelengths of travel. The selection of a time-marching method is not quite as critical, since the computational work varies linearly with the time step size. Consequently, the cost of reducing the time step is much less than the cost of reducing the $P P W$, and, furthermore, has no memory implications. Adams-Bashforth and low-storage Runge-Kutta methods can be used, with the latter often preferable because of their reduced memory requirements. Optimization of the time-marching method is generally not particularly beneficial.

For appropriate problems, such as those involving electromagnetic waves or acoustic waves in a quiescent medium, staggered spatial schemes perform very well. These can be combined with either a high-order time-marching method or the staggered leapfrog method. In the latter case, a low Courant number should be used. For large propagation distances, higher-order time-marching methods are more efficient.

The grid requirements of the explicit fourth-order methods involving simultaneous spacetime discretization are reasonably low considering the low cost of these schemes, especially the two-step explicit scheme. This suggests that sixth-order extensions of these schemes are worthy of investigation. Optimized forms of these schemes can be considered as well.

\section{A Numerical Example}

In this section, we present a numerical simulation of the propagation and reflection of an electromagnetic wave which demonstrates some of the issues discussed. Further details are available in Ref. [18]. The governing equations are the transverse magnetic set of the twodimensional time-domain Maxwell equations. The simulation consists of a pulsed plane wave incident on a perfectly-conducting cylinder. A grid containing 5,400 nodes is shown in Figure 14. Figure 15 shows a snapshot of the electric field intensity computed on a grid with 21,600 nodes using the maximum-order version of the method of Zingg et al. $[15,16]$ The dashed contours indicate negative values of the electric field intensity. This solution is visually indistinguishable from that computed using the same method on a grid with 16 times as many nodes, which is used as a baseline to estimate numerical errors.

Figures. 16 and 17 show the $L_{2}$ norm of the numerical error versus the number of grid nodes and the CPU effort, respectively. Four methods are included, the maximumorder (MO) and optimized (O10) schemes of Zingg et al. [15, 16], as well as second- (C2) and fourth-order $(\mathrm{C} 4)$ centered differences. The second- and fourth-difference schemes are combined with fourth-order Runge-Kutta time marching and include a very small amount of artificial dissipation for stability. These figures show that the higher-order and optimized methods lead to substantial reductions in both memory and CPU time. The benefits of the 


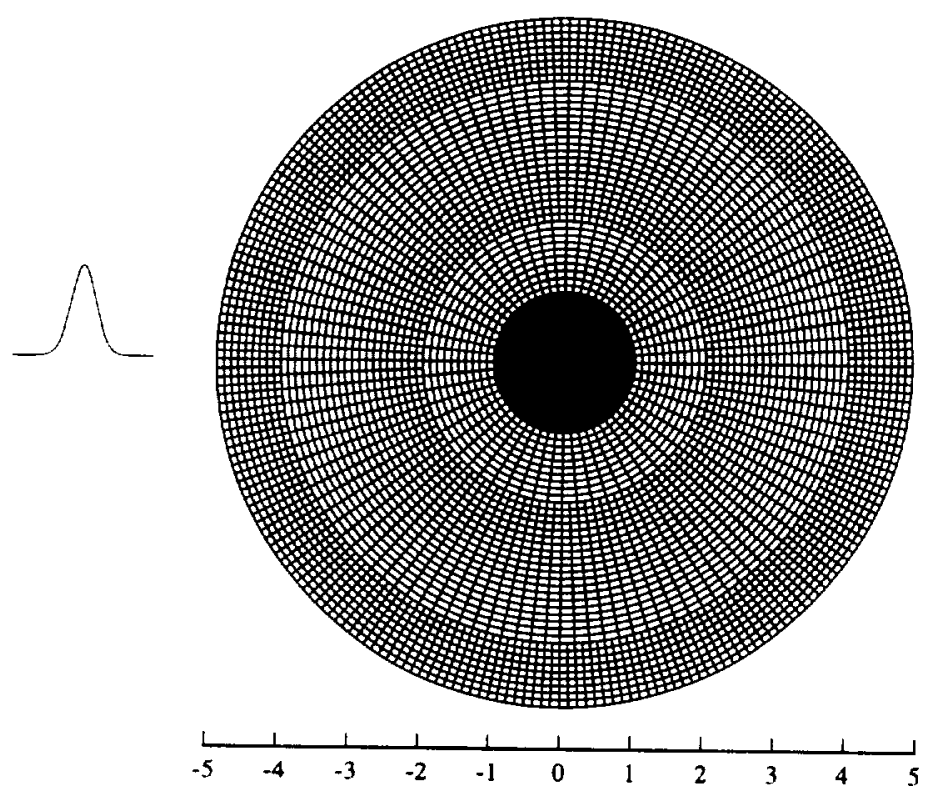

Figure 14: Grid for the perfectly-conducting cylinder.

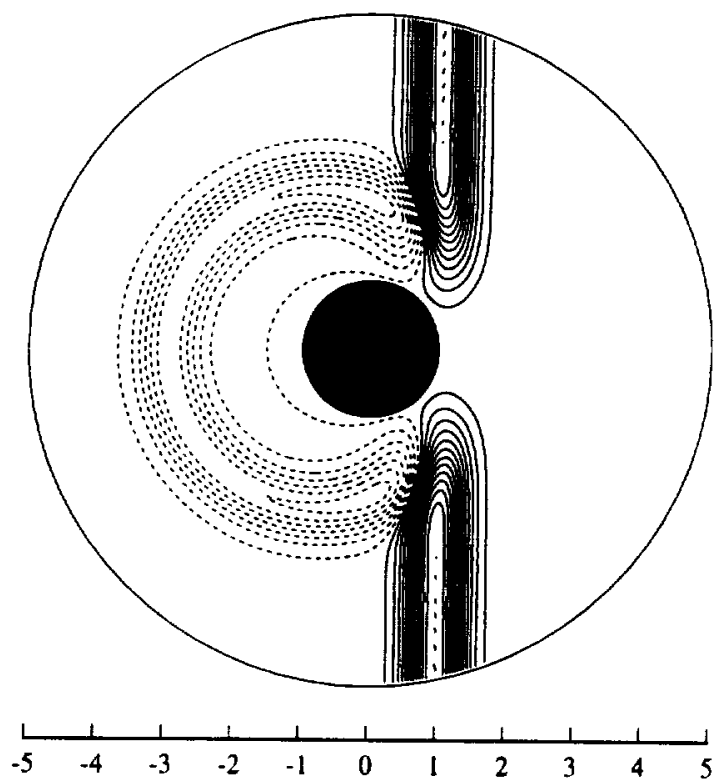

Figure 15: Computed contours of electric field intensity. 


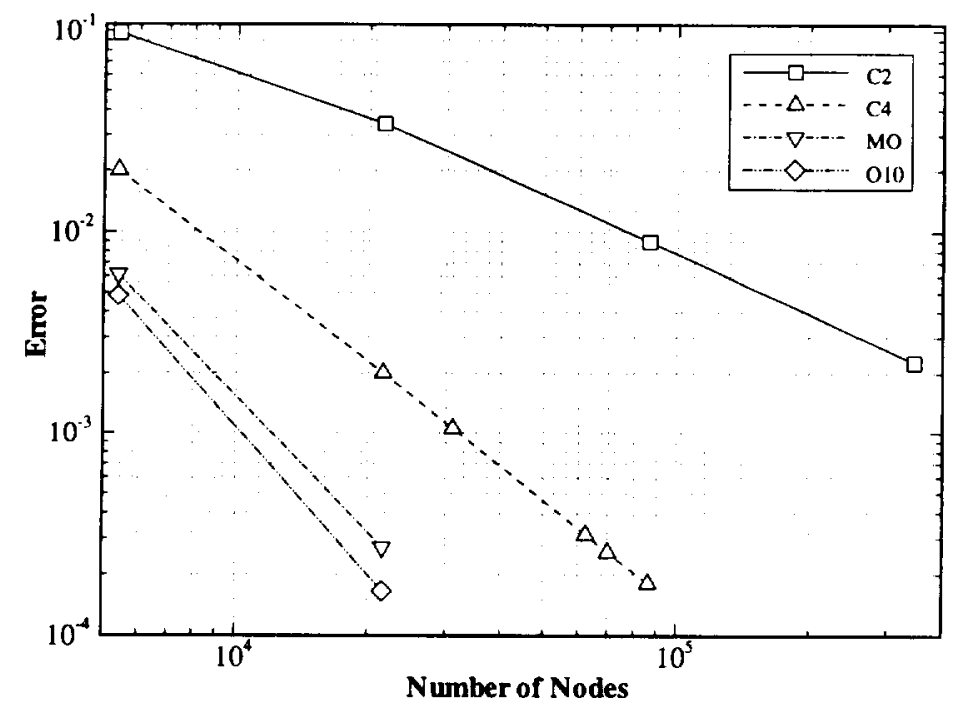

Figure 16: Error in the electric field intensity as a function of the number of grid nodes.

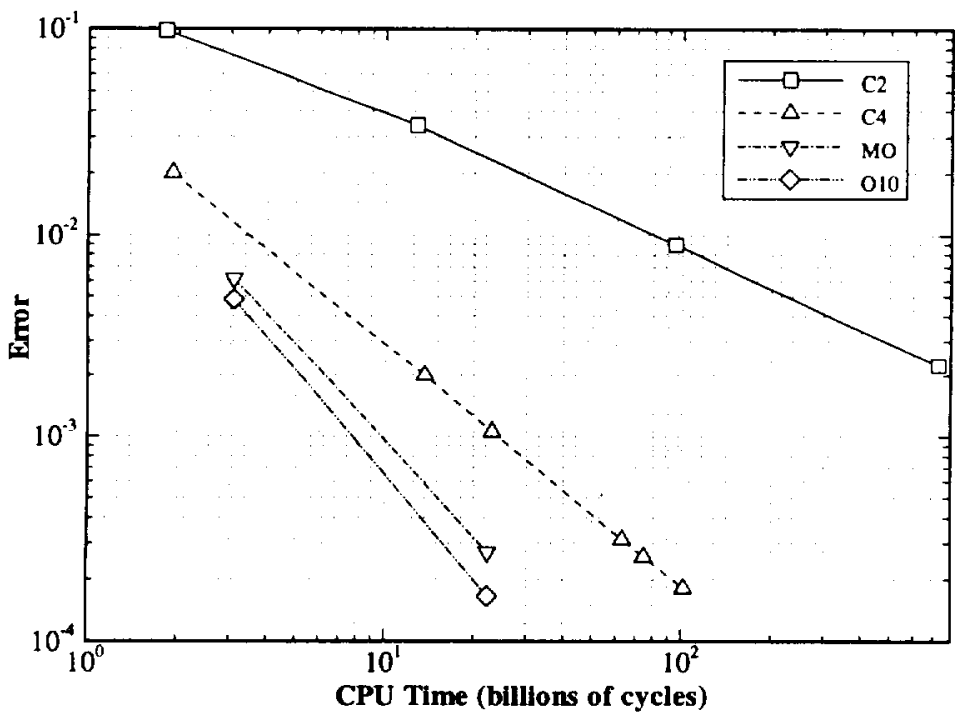

Figure 17: Error in the electric field intensity as a function of the CPU time. 
optimized scheme over the maximum-order scheme are fairly modest due to the nature of the waveform, which is a Gaussian and thus has considerable low-wavenumber content.

\section{Conclusions}

Based on the results presented, it is clear that high-order and optimized finite-difference methods will play an important role in the simulation of high frequency linear wave phenomena. Several of the methods studied have the potential to reduce substantially the computational requirements, including both CPU time and memory, for accurate simulations. Further study is required to determine the relative advantages of these methods in a practical context. Development of stable and accurate numerical boundary schemes remains a pacing item in the application of such methods. Further effort is also required in the development of gridding strategies permitting high-accuracy methods to be applied to complex geometries.

\section{References}

[1] Lighthill, J., "Report on the Final Panel Discussion on Computational Aeroacoustics," ICASE Rept. No. 92-53, 1992.

[2] Taflove, A., Computational Electrodynamics: the Finite-Difference Time-Domain Method, Artech House, USA, 1995.

[3] Yee, K.S., "Numerical Solution of Initial Boundary Value Problems Involving Maxwell's Equations in Isotropic Media," IEEE Trans. Antennas and Propagation, Vol. 14, 1966, pp. 302-307.

[4] Taflove, A., "Application of the Finite Difference Time-Domain Method to Sinusoidal Steady-State Electromagnetic Penetration Problems," IEEE Trans. Electromagnetic Compatibility, Vol. 22, 1980, pp. 191-202.

[5] Jurgens, T.G., Taflove, A., Umashankar, K.R., and Moore, T.G., "Finite-Difference Time-Domain Modeling of Curved Surfaces," IEEE Trans. Antennas Propagat., Vol. 40, 1992, pp. 357-366.

[6] Fusco, M., "FDTD Algorithm in Curvilinear Coordinates," IEEE Trans. Antennas and Propagation, Vol. 38, 1990, pp. 78-89.

[7] Madsen, N.K., and Ziolowski, R.W., "A Three-Dimensional Modified Finite Volume Technique for Maxwell's Equations," Electromagnetics, Vol. 10, 1990, pp. 147-161. 
[8] Vinokur, M., and Yarrow, M., "Finite-Surface Method for the Maxwell Equations in Generalized Coordinates," AIAA Paper 93-0463, Jan. 1993.

[9] Vinokur, M., and Yarrow, M., "Finite-Surface Method for the Maxwell Equations with Corner Singularities," AIAA Paper 94-0233, Jan. 1994.

[10] Shankar, V., Mohammadian, A.H., and Hall, W.F., "A Time-Domain Finite-Volume Treatment for the Maxwell Equations," Electromagnetics, Vol. 10, 1990, pp. 127-145.

[11] Shang, J.S., "A Fractional-Step Method for the Time Domain Maxwell Equations," $J$. Comp. Phys., Vol. 118, 1995, pp. 109-119.

[12] Cangellaris, A.C., Lin. C.-C., and Mei, K.K., "Point-Matched Time-Domain Finite Element Methods for Electromagnetic Radiation and Scattering," IEEE Trans. Antennas and Propagation, Vol. 35, 1987, pp. 1160-1173.

[13] Liu, Y., "A Generalized Finite-Volume Algorithm for Solving the Maxwell Equations on Arbitrary Grids," Conf. Proc. of 10th Annual Review of Progress in Applied Computational Electromagnetics, Vol. 1, March 1994, pp. 487-494.

[14] Shang, J.S., and Gaitonde, D., "On High Resolution Schemes for Time-Dependent Maxwell Equations," AIAA Paper 96-0832, Jan. 1996.

[15] Zingg, D.W., Lomax, H., and Jurgens, H.M., "An Optimized Finite-Difference Scheme for Wave Propagation Problems," AIAA Paper 93-0459, Jan. 1993.

[16] Zingg, D.W., Lomax, H., and Jurgens, H.M., "High-Accuracy Finite-Difference Schemes for Linear Wave Propagation," SIAM J. on Scientific Computing, Vol. 17, pp. 328-346, March 1996.

[17] Jurgens, H.M., and Zingg, D.W., "Implementation of a High-Accuracy Finite-Difference Scheme for Linear Wave Phenomena," Proceedings of the International Conf. on Spectral and Higher-Order Methods, Houston, June 1995.

[18] Jurgens, H.M., and Zingg, D.W., "Numerical Solution of the Time-Domain Maxwell Equations Using High-Accuracy Finite-Difference Methods," submitted to J. Comp. Phys., June 1996.

[19] Alford, R.M., Kelly, K.R., and Boore, D.M., "Accuracy of Finite-Difference Modelling of the Acoustic Wave Equation," Geophysics, Vol. 39, 1974, pp. 834-842.

[20] Marfurt, K.J., "Accuracy of Finite-Difference and Finite-Element Modeling of the Scalar and Elastic Wave Equations," Geophysics, Vol. 49, 1984, pp. 533-549.

[21] Dablain, M.A., "The Application of High-Order Differencing to the Scalar Wave Equation," Geophysics, Vol. 51, 1986, pp. 54-66. 
[22] Gottlieb, D., and Turkel, E., "Dissipative Two-Four Methods for Time-Dependent Problems," Math. Comp., Vol. 30, No. 136, 1976, pp. 703-723.

[23] Cohen, G., and Joly, P., "Fourth Order Schemes for the Heterogeneous Acoustics Equation," Comp. Meth. in Applied Mech. and Eng., Vol. 80, 1990, pp. 397-407.

[24] Davis, S., "Low-Dispersion Finite Difference Methods for Acoustic Waves in a Pipe," J. Acoust. Soc. Am., Vol. 90, No. 5, 1991, pp. 2775-2781.

[25] Vichnevetsky, R., and De Schutter, F., "A Frequency Analysis of Finite Difference and Finite-Element Methods for Initial Value Problems," in Advances in Computer Methods for Partial Differential Equations, edited by R. Vichnevetsky, AICA/IMACS, Rutgers University, NJ, 1975, PP. 46-52.

[26] Holberg, O., "Computational Aspects of the Choice of Operator and Sampling Interval for Numerical Differentiation in Large-Scale Simulation of Wave Phenomena," Geophysical Prospecting, Vol. 35, 1987, pp. 629-655.

[27] Lele, S.K., "Compact Finite Difference Schemes with Spectral-Like Resolution," J. Comp. Phys., Vol. 103, 1992, pp. 16-42.

[28] Haras, Z., and Ta'asan, S., "Finite-Difference Schemes for Long-Time Integration," $J$. Comp. Phys., Vol. 114, 1994, pp. 265-279.

[29] Kim, J.W., and Lee, D.J., "Optimized Compact Finite Difference Schemes with Maximum Resolution," AIAA J., Vol. 34, 1996, pp. 887-893.

[30] Lockard, D.P., Brentner, K.S., and Atkins, H.L., "High Accuracy Algorithms for Computational Aeroacoustics," AIAA Paper 94-0460, Jan. 1994.

[31] Tam, C.K.W., and Webb, J.C., "Dispersion-Relation-Preserving Finite Difference Schemes for Computational Acoustics," J. Comput. Phys., Vol. 107, 1993, pp. 262-281.

[32] Sguazzero, P., Kindelan, M., and Kamel, A., "Dispersion-Bounded Numerical Integration of the Elastodynamic Equations with Cost-Effective Staggered Schemes," Computer Methods in Applied Mechanics and Engineering, Vol. 80, 1990, pp. 165-172.

[33] Zingg, D.W., and Lomax, H., "Finite-Difference Schemes on Regular Triangular Grids," J. Comp. Phys., Vol. 108, 1993, pp. 306-313.

[34] Hu, F.Q., Hussaini, M.Y., and Manthey, J.L., "Low-Dissipation and Low-Dispersion Runge-Kutta Schemes for Computational Acoustics," J. Comp. Phys., Vol. 124, 1996, pp. 177-191.

[35] Vichnevetsky, R., and Bowles, J.B., Fourier Analysis of Numerical Approximations of Hyperbolic Equations, SIAM, Philadelphia, 1982. 
[36] Liu, Y., "Fourier Analysis of Numerical Algorithms for the Maxwell Equations," $J$. Comp. Phys., Vol. 124, 1996, pp. 396-416.

[37] Zingg, D.W., and Lomax, H., "On the Eigensystems Associated with Numerical Boundary Schemes for Hyperbolic Equations," in Numerical Methods for Fluid Dynamics, M.J. Baines and K.W. Morton, eds., Clarendon Press, Oxford, pp. 471-480, 1993.

[38] Carpenter, M.H., Gottlieb, D., and Abarbanel, S., "Stable and Accurate Boundary Treatments for Compact, High-Order Finite-Difference Schemes," Applied Numerical Mathematics, Vol. 12, pp. 55-87, 1993.

[39] Carpenter, M.H., Gottlieb, D., and Abarbanel, S., "Time-Stable Boundary Conditions for Finite-Difference Schemes Solving Hyperbolic Systems: Methodology and Application to High-Order Compact Schemes," J. Comp. Phys, Vol. 111, pp. 220-236, 1994.

[40] Olsson, P., "Summation by Parts, Projections, and Stability I," Math. Comp., Vol. 64, pp. 1035-1065, 1995.

[41] Engquist, B., and Majda, A., "Absorbing Boundary Conditions for the Numerical Simulation of Waves," Math. Comp., Vol. 31, 1977, pp. 629-651.

[42] Bayliss, A., and Turkel, E., "Radiation Boundary Conditions for Wave-Like Equations," Comm. Pure Appl. Math., Vol. 23, 1980, pp. 707-725.

[43] Mur, G., "Absorbing Boundary Conditions for the Finite-Difference Approximation of the Time-Domain Electromagnetic Field Equations," IEEE Trans. Electromagnetic Compatibility, Vol. 23, 1981, pp. 377-382.

[44] Moore, T.G., Blaschak, J.G., Taflove, A., and Kriegsmann, G.A., "Theory and Application of Radiation Boundary Operators," IEEE Trans. Antennas and Propagation, Vol. 36, 1988, pp. 1797-1812.

[45] Givoli, D., "Non-Reflecting Boundary Conditions," J. Comp. Phys., Vol. 94, 1991, pp. $1-29$.

[46] Tam, C.K.W., and Webb, J.C., "Radiation Boundary Condition and Anisotropy Correction for Finite Difference Solutions of the Helmholtz Equation," J. Comput. Phys., Vol. 113, 1994, pp. 122-133.

[47] Berenger, J.-P., "A Perfectly Matched Layer for the Absorption of Electromagnetic Waves," J. Comp. Phys., Vol. 114, 1994, pp. 185-200.

[48] Hixon, R., Shih, S.-H., and Mankbadi, R.R., "Evaluation of Boundary Conditions for Computational Aeroacoustics," AIAA J., Vol. 33, 1995, pp. 2006-2012.

[49] Tam, C.K.W., "Computational Aeroacoustics: Issues and Methods," AIAA Paper 950677, Jan. 1995. 
[50] Kurbatskii, K.A., and Tam, C.K.W., "Curved Wall Boundary Conditions for HighOrder Finite Difference Schemes in Computational Aeroacoustics," Bulletin of the American Physical Society, Vol. 39, Nov. 1994.

[51] Zingg, D.W., and Chisholm, T.T., "Runge-Kutta Methods for Linear Problems," AIAA Paper 95-1756, June 1995.

[52] Zingg, D.W., and Lomax, H., "Some Aspects of High-Order Numerical Solutions of the Linear Convection Equation with Forced Boundary Conditions," AIAA Paper 93-3381, July 1993.

[53] Zingg, D.W., "On Finite-Difference Methods for Simulating Linear Acoustic Wave Propagation," AIAA Paper 96-0280, Jan. 1996.

[54] Shubin, G.R., and Bell, J.B., "A Modified Equation Approach to Constructing Fourth Order Methods for Acoustic Wave Propagation," SIAM J. on Scientific and Statistical Computing, Vol. 8, 1987.

[55] Thomas, J.P., and Roe, P.L., "Development of Non-Dissipative Numerical Schemes for Computational Aeroacoustics," AIAA Paper 93-3382, July 1993.

[56] Zingg, D.W., Epstein, E.M., and Jurgens, H.M., "A Comparison of Finite-Difference Schemes for Computational Aeroacoustics," AIAA Paper 95-0162, Jan. 1995.

\section{Appendix}

The following are the coefficients of the finite-difference schemes studied.

The spatial operator of Haras and Ta'asan is given in eq. 3. The schemes shown in Figure 3 have $\beta=c=0$. The remaining coefficients are:

Scheme A:

$$
\begin{aligned}
\alpha & =0.3534620453 \\
a & =1.566965775 \\
b & =0.1399583152
\end{aligned}
$$

Scheme B:

$$
\begin{aligned}
\alpha & =0.3461890571 \\
a & =1.5633098070 \\
b & =0.1290683071
\end{aligned}
$$


Scheme C:

$$
\begin{aligned}
\alpha & =0.3427812069 \\
a & =1.5614141543 \\
b & =0.124148259
\end{aligned}
$$

In Figure 4, the pentadiagonal 7-point scheme has

$$
\begin{aligned}
& \alpha=0.5801818925 \\
& \beta=0.0877284887 \\
& a=1.3058941939 \\
& b=0.9975884963 \\
& c=0.0323380724
\end{aligned}
$$

The tridiagonal 7-point scheme has

$$
\begin{aligned}
& \alpha=0.3904091387 \\
& \beta=0 \\
& a=1.5638887738 \\
& b=0.2348222711 \\
& c=-0.0178927675
\end{aligned}
$$

The tridiagonal 5-point operator is scheme B above.

In Figure 5, the scheme of Lockard et al. is the average of two schemes with the following coefficients, as defined in eq. 6 :

$$
\begin{aligned}
a_{-4} & =0.0207860419 \\
a_{-3} & =-0.1500704734 \\
a_{-2} & =0.5234309723 \\
a_{-1} & =-1.34207332539 \\
a_{0} & =0.574548248808 \\
a_{1} & =0.4090357053658 \\
a_{2} & =-0.035657169508
\end{aligned}
$$

and

$$
\begin{aligned}
& a_{0}=0 \\
& a_{1}=-a_{-1}=0.763289242273 \\
& a_{2}=-a_{-2}=-0.160631393818 \\
& a_{3}=-a_{-3}=0.019324515121
\end{aligned}
$$


The optimized scheme of Zingg et al. is given by eqs. 4 and 5 with the following coefficients:

$$
\begin{aligned}
& a_{1}=0.75996126 \\
& a_{2}=-0.15812197 \\
& a_{3}=0.018760895 \\
& d_{0}=0.1 \\
& d_{1}=-0.076384622 \\
& d_{2}=0.032289620 \\
& d_{3}=-0.0059049989
\end{aligned}
$$

Tam's scheme is obtained from eq. 4 with

$$
\begin{aligned}
& a_{1}=0.770882380518 \\
& a_{2}=-0.166705904415 \\
& a_{3}=0.0208431427703
\end{aligned}
$$

The five-stage Runge-Kutta method shown in Figures. 7 and 8 has the following characteristic polynomial:

$$
\sigma(\lambda h)=1+\lambda h+\frac{(\lambda h)^{2}}{2}+\frac{(\lambda h)^{3}}{6}+\frac{(\lambda h)^{4}}{24}+\frac{(\lambda h)^{5}}{120}
$$

The six-stage method is obtained from eq. 8 with $\alpha_{5}=1 / 2, \alpha_{4}=1 / 3, \alpha_{3}=1 / 4, \alpha_{2}=1 / 5$, $\alpha_{1}=1 / 6$, leading to the following characteristic polynomial:

$$
\begin{aligned}
\sigma(\lambda h)= & 1+\lambda h+\frac{(\lambda h)^{2}}{2}+\frac{(\lambda h)^{3}}{6}+\frac{(\lambda h)^{4}}{24} \\
& +\frac{(\lambda h)^{5}}{120}+\frac{(\lambda h)^{6}}{720}
\end{aligned}
$$

The characteristic polynomial of the optimized five-stage Runge-Kutta method of Haras and Ta'asan used in Figure 9 is:

$$
\begin{aligned}
\sigma(\lambda h)= & 1+\lambda h+\frac{(\lambda h)^{2}}{2}+0.166407(\lambda h)^{3} \\
& +0.0409525(\lambda h)^{4}+0.0074510(\lambda h)^{5}
\end{aligned}
$$

The optimized temporal operator of Zingg et al. is obtained from eq. 8 with the following coefficients:

$$
\begin{aligned}
& \alpha_{1}=0.168850 \\
& \alpha_{2}=0.197348 \\
& \alpha_{3}=0.250038 \\
& \alpha_{4}=0.333306 \\
& \alpha_{5}=0.5
\end{aligned}
$$


The resulting characteristic polynomial is

$$
\begin{aligned}
\sigma(\lambda h)= & 1+\lambda h+\frac{(\lambda h)^{2}}{2}+0.16665295(\lambda h)^{3} \\
& +0.041669557(\lambda h)^{4}+0.0082233848(\lambda h)^{5} \\
& +0.0013885169(\lambda h)^{6}
\end{aligned}
$$

The scheme of Davis is obtained from eq. 10 with

$$
\begin{aligned}
& a_{0}=b_{0}=-2(C-2)(C+2) \\
& a_{1}=b_{2}=(C-1)(C-2) \\
& a_{2}=b_{1}=(C+1)(C+2)
\end{aligned}
$$

where $C=a h / \Delta x$ is the Courant number. 
